\title{
Buprenorphine/naloxone induction in a Canadian emergency department with rapid access to community-based addictions providers
}

\author{
Tina $\mathrm{Hu}, \mathrm{MD}, \mathrm{MSc}^{*}$; Melissa Snider-Adler, MD+; Larry Nijmeh, MD*†; Adam Pyle, MD*†
}

\begin{abstract}
CLINICIAN'S CAPSULE
What is known about the topic?

Opportunities to initiate buprenorphine/naloxone and facilitate addictions referrals are often missed for emergency department (ED) patients presenting in opioid withdrawal.

What did this study ask?

In an ED program offering buprenorphine/naloxone and community-based addictions follow-up for opioid use disorder, what are six-month retention rates?

What did this study find?

Approximately $88 \%$ of patients consented to ED-initiated treatment/referral, and $37 \%$ continued treatment six months after their initial visit.

Why does this study matter to clinicians?

Local implementation of a similar program using existing community resources could lead to better care for patients presenting with opioid use disorder.
\end{abstract}

\section{ABSTRACT}

Objectives: Opioid-related emergency department (ED) visits have increased significantly in recent years. Our objective was to evaluate an ED-initiated buprenorphine/naloxone program, which provided rapid access to an outpatient community-based addictions clinic, for patients in opioid withdrawal. Methods: A retrospective chart review was completed within a health system encompassing four community EDs in Ontario, Canada. Patients were screened for opioid withdrawal between April 2017-December 2017 and offered buprenorphine/naloxone treatment and referral to outpatient addictions follow-up. The main outcome measure was treatment retention in the six-month period after the index visit.

Results: The overall sample $(\mathrm{N}=49$ ) showed high healthcare utilization in the year prior to the index ED visit. $88 \%$ of patients
( $n=43$ ) consented to ED-initiated buprenorphine/naloxone and were referred to outpatient addictions follow-up, with $54 \%$ attending the initial follow-up visit. In the 6-month follow-up period from the index ED visit, $35 \%$ of patients were receiving ongoing buprenorphine/naloxone treatment and $2.3 \%$ were weaned off opioids. Patients with ongoing treatment had significantly lower number of ED visits at 3 and 6 months ( 3 and 10, respectively) compared to patients who did not show up for outpatient follow-up $(28,40)$ or started/ stopped treatment $(23,41)$.

Conclusions: Screening for opioid use disorder in the ED and initiating buprenorphine/naloxone treatment with rapid referral to an outpatient community-based addictions clinic led to a 6-month treatment retention rate of $37 \%$ and a significant reduction in ED visits at 3 and 6 months. Buprenorphine/naloxone initiation in the ED appears to be an effective intervention, but further research is needed.

\section{RÉSUMÉ}

Objectif: Le nombre de consultations aux services des urgences (SU) motivées par l'usage des opioïdes a augmenté de façon importante au cours des dernières années. L'étude visait à évaluer l'efficacité d'un programme de traitement des troubles afférents par la buprénorphine (BPN) et la naloxone, entrepris au SU et suivi d'un accès rapide à des services communautaires de consultation externe pour le traitement de la dépendance chez les patients présentant un syndrome de sevrage aux opioïdes.

Méthode: Il s'agit d'un examen rétrospectif de dossiers médicaux, réalisé dans un réseau de santé constitué $4 \mathrm{SU}$ communautaires, en Ontario, au Canada. Les patients présentant des symptômes de sevrage aux opioïdes ont d'abord été repérés entre avril 2017 et décembre 2017, puis se sont vu offrir un traitement par la BUP et la naloxone avec aiguillage vers un service de consultation externe pour le suivi. Le critère d'évaluation principal consistait en la poursuite du traitement

From *Department of Family and Community Medicine, University of Toronto, Toronto, ON; †Department of Family Medicine, Queen's University, Kingston, ON; and the ‡Department of Emergency Medicine and Critical Care, Lakeridge Health, Oshawa, ON.

Correspondence to: Dr. Tina Hu, 61 Queen Street East, $3^{\text {rd }}$ floor, Toronto, ON M5C 2T2; Email: tina.hu@mail.utoronto.ca

(C) Canadian Association of Emergency Physicians 
au cours de la période de 6 mois suivant la consultation de référence.

Résultats: L'analyse de l'échantillon global $(n=49)$ a révélé une forte utilisation des services de santé au cours de l'année précédant la consultation de référence au SU. Dans I'ensemble, $88 \%$ des patients $(n=43)$ ont accepté l'offre de traitement entrepris au SU, puis ont été dirigés vers un service de consultation externe pour le suivi; $54 \%$ de ces derniers sont allés à la première consultation. Durant le suivi de 6 mois après la consultation de référence au SU, 35\% des patients étaient encore en traitement et 2,3\% des participants étaient sevrés. Les patients encore fidèles au traitement ont connu un nombre significativement moins élevé de consultations au SU au bout de 3 mois et de 6 mois ( 3 et 10, respectivement) que les patients qui ne sont pas présentés au service de consultation externe
$(28,40)$ ou qui ont entrepris le traitement mais qui ne l'ont pas poursuivi $(23,41)$.

Conclusion: Le dépistage des troubles liés à l'usage des opioïdes, au SU, et l'instauration du traitement par la BUP et la naloxone avec aiguillage rapide vers un service communautaire de consultation externe pour le traitement de la dépendance se sont traduits par un taux de rétention des patients de $37 \%$ au bout de 6 mois et par une réduction significative du nombre de consultations au SU au bout de 3 mois et de 6 mois. L'instauration du traitement au SU semble donc une intervention efficace, mais il faudrait poursuivre la recherche sur le sujet.

Keywords: Addiction medicine, emergency medicine, primary care

\section{INTRODUCTION}

Canada is amid an opioid crisis, with unintentional drug overdoses becoming a public health crisis. It is estimated that there were 3,998 apparent opioid-related deaths in Canada in 2017, with no regions spared: $76 \%$ of opioid-related deaths occurred in males, $27 \%$ in individuals between 30 and 39 years of age, and an increasing amount of opioid-related deaths (72\%) involved fentanyl. ${ }^{1,2}$ The pervasiveness of the opioid crisis is highlighted by the fact that opioid-related deaths are increasing in all age groups, both sexes, and all income brackets. ${ }^{1}$

Based on recent Canadian guidelines, buprenorphine/ naloxone is considered first-line for opioid use disorder (OUD) to reduce the risk of toxicity, morbidity, and mortality. ${ }^{3}$ Compared with methadone, buprenorphine/naloxone has a milder adverse effect profile, requires a shorter time (1-3 days) to achieve therapeutic dose, and has a lower risk of toxicity and drug interactions. $^{3}$ Both buprenorphine/naloxone and methadone treatments are associated with reduced all-cause mortality and should be considered for all patients with OUD. ${ }^{4}$ Previous data have shown that only $10.8 \%$ of patients with substance use disorder receive specialty treatment annually. ${ }^{5}$ To address this, brief interventions incorporating both behavioural and medical treatment in clinical settings such as the emergency department (ED), with a bridge to follow-up therapy, have been promoted for treating substance use disorders.

Patients with OUD are frequently seen in EDs for reasons such as opioid overdose, withdrawal, skin and soft tissue infections, endocarditis, osteomyelitis, and comorbid mental health issues. In many EDs, the primary option for managing OUD is passive referral to outpatient addiction clinics, where treatment with methadone or buprenorphine/naloxone would be started. A randomized controlled trial in the United States has suggested that patients with OUD are more likely to continue treatment if opioid agonist therapy is started in the ED than if they are only referred for treatment. ${ }^{6}$ Buprenorphine/naloxone is particularly wellsuited for initiation in the ED setting because it is effective, easy to prescribe, and allows for safe home induction for patients who are not yet in withdrawal. ${ }^{7}$

There has been limited research on the initiation of buprenorphine/naloxone in the ED setting, with the major studies based in the United States. The goal of this study was to evaluate an ED-initiated buprenorphine/naloxone program with referral to an existing community-based addictions clinic, for patients in opioid withdrawal, in a Canadian context.

\section{METHODS}

This retrospective chart review study was approved by the institutional ethics review board of Lakeridge Health (Oshawa, ON). Lakeridge Health is a health system that encompasses four acute care community hospitals with EDs (Bowmanville, Oshawa, Port Perry, and Ajax/Pickering).

\section{Program description}

In this program, multiple training sessions for screening for opioid withdrawal and OUD and administering/ 
prescribing buprenorphine/naloxone were given to ED health care providers. Patients in the ED between April 2017 and December 2017 were screened for OUD by health care providers if they presented to the ED with signs of opioid withdrawal or were opioidseeking. To be eligible for the program, patients had to meet criteria for OUD using Diagnostic and Statistical Manual-5 (DSM-5) criteria and deemed as being in at least mild opiate withdrawal as assessed with the Clinical Opiate Withdrawal Scale ${ }^{8}$ (COWS; a score greater than 5; refer to Appendix 1). Patients who had used opioids within the previous 12 hours or who were on methadone were not eligible for buprenorphine/naloxone treatment. At the time of this study, ED-specific protocols for the initiation of buprenorphine/naloxone were not available. Our protocol was developed and agreed by consensus among both ED and addictions health care providers (refer to Appendix 2 for the treatment algorithm). Patients who were eligible for and consented to treatment were given an initial dose of buprenorphine/ naloxone in the ED $(2-4 \mathrm{mg})$ and were witnessed by a nurse to ensure the medication was taken sublingually and had fully dissolved. Patients were re-assessed in 1-2 hours using the COWS score. If still in withdrawal, another $2-4 \mathrm{mg}$ of buprenorphine/naloxone was administered sublingually based on the severity of withdrawal symptoms. The maximum dose administered in the ED was $8 \mathrm{mg}$. The patient was then discharged from the ED with a package containing information on opioid withdrawal symptoms, options for managing opioid withdrawal, contact information for clinics prescribing methadone and buprenorphine/naloxone, and information regarding outpatient case management programs. A buprenorphine/naloxone prescription with up to three daily observed doses, a request to the pharmacy to provide take-home naloxone, and referral were faxed to the rapid access addiction clinic (RAAC). Patients were advised to go to the RAAC the next day and make an appointment on the same day or next business day. The prescription was either filled at the pharmacy on-site with the RAAC or sent to another pharmacy of their choice.

\section{Data collection}

Charts of patients who were enrolled in the study were reviewed retrospectively (refer to Appendix 3 for the selection algorithm). Demographic information (age, sex, and housing status), information regarding substance use (previous rehabilitation for OUD, substance use other than opioids, concurrent psychiatric comorbidity, number of ED visits in the previous year, and percentage of ED visits related to substance use) were collected. We determined the number of referred patients who were seen in the RAAC post-ED discharge; average number of days from ED discharge to first RAAC appointment; ongoing RAAC follow-up and opioid agonist treatment at one, three, and six months postindex ED visit (confirmed with treatment providers); number of ED visits at one, three, and six months postindex ED visit (and percentage of visits related to substance use); and number of hospitalizations at one, three, and six months post-index ED visit (and percentage of hospitalizations related to substance use).

\section{Statistical Analysis}

All data were anonymized before analysis. Descriptive statistics, chi-square tests, and Kruskal-Wallis $\mathrm{H}$ tests were used to analyze the data. A $p$-value of less than 0.05 was used to determine significance. Statistical analyses were completed using SPSS (version 20).

\section{RESULTS}

The overall sample included 49 patients (mean age 37.0 years, standard deviation $[\mathrm{SD}]=12.3$ ). Fifty-seven percent of the study sample was male. Baseline patient characteristics for the overall sample are shown in Table 1. Of note, $24 \%$ of the overall sample had previous rehabilitation or supportive treatment for OUD, and $71 \%$ had at least one ED visit in the previous year (total of 119 visits among the sample, with $57 \%$ of the visits related to substance use). Approximately $45 \%$ had a concurrent psychiatric comorbidity, and $24 \%$ reported substance use other than opioids. Approximately $88 \%$ of these patients $(n=43)$ consented to receive buprenorphine/ naloxone treatment in the ED and referral to an outpatient RAAC. Excluded patients included: patients who consented to only receive buprenorphine/naloxone in the ED for withdrawal management and not referral $(6.1 \%)$; and patients who consented to referral only (6.1\%).

Data regarding outpatient RAAC follow-up and postindex ED visit health care utilization for patients in the program are shown in Table 2. Approximately 54\% of patients referred for outpatient RAAC follow-up 


\begin{tabular}{|lc|}
\hline Table 1. Overall patient characteristics & \\
\hline & $\begin{array}{c}\text { Overall sample } \\
(N=49)\end{array}$ \\
\hline Mean (SD) age, years & $37.0(12.3)$ \\
Male sex, \% & $28(57 \%)$ \\
Previous rehabilitation or supportive treatment for & $12(24 \%)$ \\
$\quad$ opioid use disorder & \\
Patients with ED visits in the previous year, \% & $35(71 \%)$ \\
Total number of ED visits & 119 \\
Total ED visits related to substance use, \% & $68(57 \%)$ \\
$\quad$ Mean number of ED visits in the previous year & $2.4(3.1)$ \\
(SD) & $20(41 \%)$ \\
Patients with one or more previous ED visits & \\
related to substance use & $7(14 \%)$ \\
Unstable housing status, \% & $12(24 \%)$ \\
Substance use other than opioids, \% & $22(45 \%)$ \\
Concurrent psychiatric comorbidity, $\%$ & $43(88 \%)$ \\
Buprenorphine/naloxone given in ED and referral & \\
to outpatient addictions clinic, \% & $5.0(2.4)$ \\
Total mean (SD) buprenorphine/naloxone dose in & \\
ED, mg & \\
\hline ED = emergency department; SD = standard deviation. & \\
\hline
\end{tabular}

attended the initial appointment. The mean number of days from ED discharge until the first outpatient RAAC follow-up was 2.7 days $(\mathrm{SD}=2.9)$. The mean age of this group was 37 years $(\mathrm{SD}=12)$. At six months post-index ED visit, 35\% had ongoing buprenorphine/ naloxone treatment, $2.3 \%$ were weaned successfully off opioids, $16 \%$ started/stopped buprenorphine/naloxone treatment, and $47 \%$ did not show to an initial appointment.

Table 3 shows the differences in health care utilization post-index ED visit between patients who continued with buprenorphine/naloxone treatment/weaned successfully off opioids compared with patients who started/stopped treatment or did not show up to the initial RAAC appointment. Between groups, there were no significant differences in terms of age (Kruskal-Wallis $\mathrm{H}$ test $\chi^{2}(2)=1.03, p=0.60$ ), sex (chi-square test $\chi^{2}(2)=$ 4.77, $p=0.09)$, previous rehabilitation or supportive treatment for OUD (chi-square test $\chi^{2}(2)=1.49, p=$ 0.47 ), housing status (chi-square test $\chi^{2}(2)=0.11, p=$ 0.95 ), use of other substances than opioids (chi-square test $\left.\chi^{2}(2)=1.01, p=0.60\right)$, comorbid psychiatric conditions (chi-square test $\chi^{2}(2)=2.67, p=0.26$ ), and number of ED visits in the previous year (Kruskal-Wallis $\mathrm{H}$ test $\left.\chi^{2}(2)=5.46, p=0.07\right)$. A significantly lower proportion of patients in the ongoing treatment group had at least one

\begin{tabular}{|c|c|}
\hline & $\begin{array}{l}\text { Sample } \\
(n=43)\end{array}$ \\
\hline Seen at outpatient addictions clinic, $\%$ & $23(54 \%)$ \\
\hline $\begin{array}{l}\text { Patients with ED visits one month after index ED } \\
\text { visit, \% }\end{array}$ & $14(33 \%)$ \\
\hline Total number of ED visits & 24 \\
\hline Mean number of ED visits (SD) & $0.56(1.2)$ \\
\hline ED visits related to substance use, \% & $18(75 \%)$ \\
\hline $\begin{array}{l}\text { Patients with ED visits three months after index ED } \\
\text { visit, \% }\end{array}$ & $20(47 \%)$ \\
\hline Total number of ED visits & 54 \\
\hline Mean number of ED visits (SD) & $1.3(2.7)$ \\
\hline ED visits related to substance use, $\%$ & $39(72 \%)$ \\
\hline $\begin{array}{l}\text { Patients with ED visits six months after index ED } \\
\text { visit, \% }\end{array}$ & $25(58 \%)$ \\
\hline Total number of ED visits & 91 \\
\hline Mean number of ED visits (SD) & $2.2(4.3)$ \\
\hline ED visits related to substance use, \% & $55(60 \%)$ \\
\hline $\begin{array}{l}\text { Patients with hospitalization one month after index } \\
\text { ED visit, \% }\end{array}$ & $3(7.0 \%)$ \\
\hline Total number of hospitalizations & 3 \\
\hline $\begin{array}{l}\text { Hospitalizations related to substance use or } \\
\text { psychiatric issue, \% }\end{array}$ & $3(100 \%)$ \\
\hline Mean (SD) length of hospitalization, days & $2.0(1)$ \\
\hline $\begin{array}{l}\text { Patients with hospitalization three months after index } \\
\text { ED visit, \% }\end{array}$ & $3(7.0 \%)$ \\
\hline Total number of hospitalizations & 5 \\
\hline $\begin{array}{l}\text { Hospitalizations related to substance use or } \\
\text { psychiatric issue, \% }\end{array}$ & $5(100 \%)$ \\
\hline Mean (SD) length of hospitalization, days & $9.3(18)$ \\
\hline $\begin{array}{l}\text { Patients with hospitalization six months after index } \\
\text { ED visit, \% }\end{array}$ & $9(21 \%)$ \\
\hline Total number of hospitalizations & 14 \\
\hline $\begin{array}{l}\text { Hospitalizations related to substance use or } \\
\text { psychiatric issue, \% }\end{array}$ & $12(86 \%)$ \\
\hline Mean (SD) length of hospitalization, days & $5.0(11)$ \\
\hline \multicolumn{2}{|l|}{ Outcome six months after index ED visit } \\
\hline Ongoing buprenorphine/naloxone treatment & $15(35 \%)$ \\
\hline Weaned successfully off opioids & $1(2.3 \%)$ \\
\hline $\begin{array}{l}\text { Started and stopped buprenorphine/naloxone } \\
\text { treatment }\end{array}$ & 7 (16\%) \\
\hline No show to initial appointment or after assessment & $20(47 \%)$ \\
\hline
\end{tabular}

ED visit at three months post-index ED visit (chi-square test $\chi^{2}(2)=8.17, p=0.02$ ). Patients in the ongoing treatment group had significantly fewer total ED visits at three months (Kruskal-Wallis $H$ test $\chi^{2}(2)=9.98$, $p=0.01)$, with a mean rank total number of ED visits 


\begin{tabular}{|c|c|c|c|c|}
\hline & $\begin{array}{l}\text { No show to } \\
\text { RAAC appointment } \\
(n=20)\end{array}$ & $\begin{array}{l}\text { Started and } \\
\text { stopped treatment } \\
(n=7)\end{array}$ & $\begin{array}{l}\text { Ongoing treatment or } \\
\text { weaned successfully } \\
(n=16)\end{array}$ & $p$-value \\
\hline ED visits one month after index visit, \% & $21 \%$ & $43 \%$ & $13 \%$ & 0.10 \\
\hline Number of total ED visits & 12 & 10 & 2 & 0.08 \\
\hline Patients with ED visits related to substance use, \% & $40 \%$ & $29 \%$ & $6.2 \%$ & 0.07 \\
\hline ED visits three months after the index visit, \% & $60 \%$ & $71 \%$ & $19 \%$ * & $0.02 *$ \\
\hline Number of total ED visits & 28 & 23 & $3^{*}$ & $0.01 *$ \\
\hline Patients with ED visits related to substance use, \% & $55 \%$ & $29 \%$ & $6.2 \% *$ & $0.01 *$ \\
\hline ED visits six months after the index visit, \% & $65 \%$ & $86 \%$ & $38 \%$ & 0.07 \\
\hline Number of total ED visits & 40 & 41 & $10^{*}$ & $0.02 *$ \\
\hline Patients with ED visits related to substance use, \% & $60 \%$ & $43 \%$ & $13 \% *$ & $0.02 *$ \\
\hline Patients with hospitalization one month after index ED visit, \% & $5 \%$ & $14 \%$ & $6.2 \%$ & 0.70 \\
\hline Number of hospitalizations & 1 & 1 & 1 & 0.71 \\
\hline $\begin{array}{l}\text { Patients with hospitalization three months after index ED } \\
\text { visit, \% }\end{array}$ & $5 \%$ & $14 \%$ & $6.2 \%$ & 0.70 \\
\hline Number of hospitalizations & 2 & 2 & 1 & 0.69 \\
\hline Patients with hospitalization six months after index ED visit, \% & $30 \%$ & $29 \%$ & $6.2 \%$ & 0.19 \\
\hline Number of hospitalizations & 7 & 4 & 3 & 0.25 \\
\hline
\end{tabular}

of 15 for the treated group, 29 for the group that started/ stopped treatment, and 25 for the group that did not show up to the RAAC appointment. Further, patients in ongoing treatment had significantly fewer total number of ED visits at six months (Kruskal-Wallis $\mathrm{H}$ test $\left.\chi^{2}(2)=7.99, p=0.02\right)$, with a mean rank total number of ED visits of 16 for the treated group, 31 for the group that started/stopped treatment, and 24 for the group that did not show up to the RAAC appointment. A significantly lower proportion of the ongoing treatment group had ED visits related to substance use at three months (chi-square test $\chi^{2}(2)=9.68, p=0.01$ ) and six months post-index ED visit (chi-square test $\chi^{2}(2)=$ $8.43, p=0.02$ ). There were no significant differences between groups in terms of total number of ED visits at one month (Kruskal-Wallis $H$ test $\chi^{2}(2)=5.16$, $p=0.08$ ), hospitalizations at one month (Kruskal-Wallis $\mathrm{H}$ test $\left.\chi^{2}(2)=0.69, p=0.71\right)$, hospitalizations at three months (Kruskal-Wallis H test $\chi^{2}(2)=0.74, p=0.69$ ), or hospitalizations at six months (Kruskal-Wallis $\mathrm{H}$ test $\left.\chi^{2}(2)=2.78, p=0.25\right)$.

\section{DISCUSSION}

To address the growing issue with OUD in the ED setting, we developed a program to screen for OUD among
ED patients presenting in opioid withdrawal, offer buprenorphine/naloxone treatment, and facilitate a referral to an outpatient community-based RAAC. Our study showed that a majority of patients presenting with OUD in the ED setting (88\%) were agreeable to starting buprenorphine/naloxone treatment and consented to referral for outpatient addictions follow-up, with $54 \%$ attending the initial appointment. Our results show that $35 \%$ of patients had ongoing buprenorphine/ naloxone treatment and $2.3 \%$ were weaned successfully off opioids six months after the index ED visit. Our study showed significantly fewer ED visits at three and six months after the index visit among patients in ongoing treatment were successfully weaned off opioids compared with those who did not attend follow-up or started and stopped treatment.

There has been limited research examining outcomes from ED-initiated buprenorphine/naloxone programs with rapid referral to outpatient addiction clinics, with most of the research conducted in the United States. D'Onofrio and colleagues conducted a randomized controlled trial in the United States to test the efficacy of: 1) screening for opioid dependence and referral to treatment; 2) screening, brief intervention, and facilitated referral to community-based treatment services; and 3) screening, brief intervention, ED-initiated treatment with buprenorphine/naloxone, 
and referral to primary care for 10 -week follow-up. ${ }^{6}$ Results showed that $78 \%$ of patients in the EDinitiated buprenorphine/naloxone group v. $37 \%$ in the referral group and $45 \%$ in the brief intervention group were engaged in treatment at one month that was a significant difference. In a follow-up analysis, D'Onofrio and colleagues showed that the ED-initiated buprenorphine/naloxone group continued to have a significantly higher proportion of patients engaged in treatment at two months, but there were no significant differences at 6 and 12 months. ${ }^{9}$ There were several key differences between our study and the study conducted in the United States: 1) we did not have a control group to assess treatment retention rates for patients who just received a referral; 2) the US study hired research associates to conduct screening for OUD in the ED with potential selection bias of patients recruited based on the time when the research associates were in the ED, and our study utilized existing resources (ED health care providers), making it more feasible in settings where funding is limited; 3) the US study was based at one urban teaching hospital ED, and our study was based at a network of four acute care community hospitals with EDs; 4) engagement in formal addiction treatment at 2, 6, and 12 months in the US study was based on self-report and not confirmed with treatment providers (prone to self-report bias), and our follow-up rates were confirmed with treatment providers; and 5) in the US study, after 10 weeks of office-based buprenorphine/naloxone treatment within the same centre, patients were transferred for ongoing maintenance treatment to either a community program/clinician or offered detoxification over two weeks. The US study showed no differences in groups after two months, which is when this transition of care occurred. This may also reflect the significant differences in the health care system between Canada and the United States. In Canada, residents are covered by public universal health insurance, with many health care services covered. Buprenorphine/naloxone is publicly funded in Canada for many patients: for example, in Ontario, coverage is provided for patients under 25 years of age, over 65 years of age, and those receiving social assistance, which greatly increases access for patients. In most Canadian provinces, there is no exemption required to prescribe buprenorphine/naloxone, which has helped reduce barriers to access, compared with the mandatory waiver in the United States. ${ }^{10}$ In the United States, most health care services are funded through private insurance, with a significant portion not having any coverage: in the US study, $22 \%$ did not have any health insurance that may have impacted treatment retention rates after 10 weeks when patients were transferred to other clinics and may not have had coverage or the ability to pay for buprenorphine/naloxone.

In addition, this study highlights a successful partnership between hospital EDs and existing buprenorphine/ naloxone prescribers and pharmacies to provide rapid access to medication-assisted treatment. Given the scarcity of system funding, this approach may provide a cost-effective solution through utilization of existing community resources and is a model that is feasible in other communities. Expanding the use of ED-initiated buprenorphine/naloxone with rapid follow-up can help increase access to treatment options for patients with OUD, a chronic and relapsing condition that has significant mortality rates when untreated. Our study adds to the literature on "interim" opioid agonist treatment, in which buprenorphine/naloxone treatment is initiated while patients await more comprehensive addiction services. Given the increasing prevalence of OUD and mortality rates, it is important to initiate programs that reduce barriers to treatment access and reduce wait times for accessing treatment.

\section{Limitations}

Limitations of this study include a relatively small sample size within a single network of community hospitals that limited the precision and generalizability of these findings and a lack of a control group to compare what treatment retention rates would be without buprenorphine/ naloxone initiation in the ED. Further research with a larger sample size, broad screening for OUD to ensure patients presenting with opioid-related complications are also included, and a longer follow-up are needed to evaluate patient outcomes fully. We were unable to follow-up with patients individually to determine reasons for not attending RAAC follow-up or for stopping buprenorphine/naloxone treatment, which would be an avenue for future research. Interventions that focus on assisting patients with navigating a complex health care system, arranging free transportation to the outpatient clinic, and addressing other social determinants of health should be considered in future research, as these factors may impact both initial follow-up and treatment retention rates. 


\section{CONCLUSIONS}

In this study examining short-term follow-up after EDinitiated buprenorphine/naloxone with referral to an outpatient RAAC for patients presenting with opioid withdrawal, approximately $37 \%$ were in ongoing maintenance therapy or weaned off opioids. Patients with ongoing treatment had significantly fewer total ED visits at three and six months compared with patients who did not show up for follow-up or started/stopped treatment, which suggests that ED-initiated buprenorphine/naloxone is an effective intervention, but further research is needed.

\section{SUPPLEMENTARY MATERIAL}

The supplementary material for this article can be found at https://doi.org/10.1017/cem.2019.24

Competing interests: None declared.

\section{REFERENCES}

1. Gomes T. Latest trends in opioid-related deaths in Ontario: 1991 to 2015: Ontario Drug Policy Research Network; 2017.
2. Government of Canada. Overview of national data on opioidrelated harms and deaths 2018. Available at: https://www. canada.ca/en/health-canada/services/substance-use/problematicprescription-drug-use/opioids/data-surveillance-research/ harms-deaths.html (accessed December 28, 2018).

3. Bruneau J, Ahamad K, Goyer MÈ, et al. Management of opioid use disorders: a national clinical practice guideline. $C M A \mathcal{7}$ 2018;190(9):E247-57.

4. Larochelle MR, Bernson D, Land T, et al. Medication for opioid use disorder after nonfatal opioid overdose and association with mortality: a cohort study. Ann Intern Med 2018;169 (3):137-45.

5. Bogenschutz MP, Donovan DM, Mandler RN, et al. Brief intervention for patients with problematic drug use presenting in emergency departments: a randomized clinical trial. 7AMA Intern Med 2014;174(11):1736-45.

6. D'Onofrio G, O'Connor PG, Pantalon MV, et al. Emergency department-initiated buprenorphine/naloxone treatment for opioid dependence: a randomized clinical trial. 7AMA 2015;313(16):1636-44.

7. Lee JD, Grossman E, DiRocco D, Gourevitch MN. Home buprenorphine/naloxone induction in primary care. 7 Gen Intern Med 2009;24(2):226-32.

8. Wesson DR, Ling W. The Clinical Opiate Withdrawal Scale (COWS). 7 Psychoactive Drugs 2003;35(2):253-9.

9. D’Onofrio G, Chawarski MC, O’Connor PG, et al. Emergency department-initiated buprenorphine for opioid dependence with continuation in primary care: outcomes during and after intervention. 7 Gen Intern Med 2017;32 (6):660-6.

10. Huhn AS, Dunn KE. Why aren't physicians prescribing more buprenorphine? 7 Subst Abuse Treat 2017;78:1-7. 